\title{
Die Krise der Finanzmärkte und die Rückkehr des Staates
}

von Harold James

Die gegenwärtige Finanzkrise lässt die Rufe nach dem Staat lauter werden, der die gegebenen Probleme lösen soll. Es stellt sich allerdings die Frage, ob daraus nicht neue Schwierigkeiten erwachsen können. Die Kreditklemme des Jahres 2008 hat in der Tat ernsthafte Schwächen des Finanzsektors offen gelegt. Die historische Erfahrung lehrt jedoch, dass solchen Problemen auf lange Sicht besser durch technologische Innovationen als durch staatliche Interventionen begegnet werden kann. Hinzu kommt, dass nur sehr wenige Staaten tatsächlich über die Kapazitäten verfügen, steuernd in globale ökonomische Zusammenhänge einzugreifen. Angesichts der jüngsten Entwicklungen erscheint eine Abkehr von der Globalisierung und eine Rückkehr zu nationalen Problemlösungsversuchen wahrscheinlich.

In the current financial crisis, calls for the state to solve the given problems rise. This might, however, lead to new difficulties. The credit crunch of the year 2008 displayed indeed serious weaknesses of the financial sector. Nevertheless, historical experience shows that such problems are rather solved by technological innovations than by state intervention. Furthermore, only very few states possess the necessary capacities for influencing global economic developments. By now, turning away from globalisation and returning to national attempts at problem-solving seems probable.

Es ist eine schwere Verpflichtung, wenn ein Wirtschaftshistoriker in der aktuellen Krise versucht, das jetzt Geschehende historisch verständlich zu machen. Mitten in einer Krise, die von Natur aus immer neue vollkommen unvorhergesehene Überraschungen bringt, suchen wir nach einem historischen Muster, das uns als Schablone für die unberechenbare Zukunft dienen könnte, und somit auch für die Formulierung von Lösungen oder Auswege aus der Krise. Auf einmal blicken wir alle wie gebannt auf die Wirtschaftsgeschichte, besonders auf die Geschichte der großen Wirtschaftskrisen: 1929 und 1931. Wir suchen nach Erfahrungen im Umgang mit Krisen. Historische Lösungen sind wieder gefragt. Doch oft sind sie im höchsten Maße problematisch. 
Am Anfang der größten aller griechischen Tragödien klagt ein alter Priester in der von einer Pest und Seuche geplagten Stadt Theben:

„Jetzt aber auch, o Haupt des Ödipus!

Stark über alle, flehen wir dich an,

Demüthig, einen Schutz zu erfinden

Habst du gehört von Göttern eine Stimme,

Habst du's von einem Manne, denn ich weiß

Dass auch Verhängnisse sogar am meisten

Sich durch den Rat Erfahrener beleben.

Wohlan, der Menschen Bester! Richte wieder auf

Die Stadt, wohlan sei klug!“

Was der Priester und die gesamte Stadt nicht wissen, ist: Der Mann, den sie um Rettung anflehen, bringt selbst die Seuche mit sich, die auf Theben lastet.

Mitten in der Finanzkrise schauen wir alle nun wie gebannt auf den Staat. Er soll uns aus der Krise erlösen. Doch könnte auch dieser Retter, wie Ödipus, mit einem Fluch beladen sein?

Ödipus schickte seinen Schwager Kreon zum Orakel nach Delphi, also zum griechischen Äquivalent der modernen Wirtschaftsberater. Die Anweisungen im alten Griechenland waren so, wie sie auch heute noch sind: unberechenbar vage und interpretationsbedürftig - also delphisch. Entsprechend waren die Politiker der griechischen Antike mit den zweideutigen Empfehlungen der Wissenschaft höchst unzufrieden. Später verhöhnt Kreon den blinden Propheten Teiresias mit den Worten: „Die Seherart liebt nämlich all das Silber.“ Heute reagieren Politiker auf die Empfehlungen der Wirtschaftswissenschaft ganz ähnlich. Neulich klagte Bundesfinanzminister Peer Steinbrück, es gebe „keine Blaupause als politische Handlungsanweisung“. „Und - ich sage es ganz offen“, so Steinbrück weiter, „die Wirtschaftswissenschaftler haben keine verlässlichen Empfehlungen für uns. Sie sind nicht minder vielstimmig und vor allem angelegt auf öffentliche Selbstdarstellung wie die Politik auch."

In diesem Beitrag werden zwei längerfristige Perspektiven zur gegenwärtigen Krise angeboten. Diese Ausblicke werden vielleicht tröstlich, womöglich sogar verharmlosend erscheinen. Am Schluss folgt eine eher kurzfristigere und eher alarmierende Interpretation. Die gegenwärtige Krise lässt sich auf drei verschiedene Arten interpretieren: Erstens als großer Strukturbruch, zweitens als eine 
Verschiebung der machtpolitischen Dynamik der Welt, und drittens schließlich als eine neue Krise der Integration und des liberalen Denkens.

\section{I.}

2008 wird als Jahr eines epochalen Wandels im Gedächtnis bleiben - eines Wandels jener weltpolitischen und weltwirtschaftlichen Ordnung, die aus der rasanten Globalisierung am Ende des 20. Jahrhunderts entstanden war. Schaut man auf die ökonomischen Daten, so sieht es für 2009 noch weitaus düsterer aus als im vergangenen Jahr. Der IWF hat gerade seine Prognose für das globale Wachstum 2009 von $2,2 \%$ auf $0,5 \%$ herabgestuft, und es kann sein, dass auch diese Zahl zu optimistisch ist. Zuvor war die Weltwirtschaft in rasantem Tempo gewachsen - seit 2001 lag die reale Wachstumskurve sogar noch wesentlich höher als die Trendlinie.

Alle historische Erfahrung verweist auf eine Korrelation zwischen überdurchschnittlichem Wachstum und der Schwere der darauf folgenden Krise. Wir haben bereits viele bedrückende Erfahrungen mit der lang anhaltenden Wirkung von Finanz- und Bankenkrisen gemacht. Dabei sind sich solche Krisen erstaunlich ähnlich - ganz gleich ob sie in Schwellenländern wie Indonesien, Korea, Thailand und Argentinien vorkommen, oder in hoch entwickelten Industrieländern. So fielen die Aktienkurse in Schweden nach 1991 um 45 \%, in Japan nach 1992 um 65 \% während einer Rezession.

Die mittleren Werte dieser historischen Daten zeugen von der lang anhaltenden Wirkung eines solchen Rückgangs. Nach der Kalkulation von Reinhart und Rogoff dauerte es im Schnitt sechs Jahre, bis sich die betroffene Wirtschaft gesundgeschrumpft hatte. Zugleich stieg die Arbeitslosigkeit in einer durchschnittlichen Spanne von vier Jahren um sieben Prozent. ${ }^{2}$ Zumindest was die Industrieländer betrifft, sind während der Nachkriegszeit solche Zusammenbrüche aber immer nur in einzelnen Staaten vorgekommen. Dagegen sehen wir uns heute mit einer allgemeinen Krise konfrontiert, die in nahezu überall gleichzeitig auftritt.

Wir stehen nun vor dem Ende jener Form der Globalisierung, die überwiegend durch eine massive Ausweitung der Kapitalströme angetrieben wurde. Sehr alte und vergessen geglaubte Rezepte werden wieder hervorgeholt. Karl Marx ist zurückgekehrt, wenn auch nicht wirklich aus dem Grab, so doch aus den ver-

2 Reinhart, C.M./Rogoff, K.S.: The Aftermath of Financial Crises, NBER Working Paper 14656, Cambridge, MA, Januar 2009. 
staubten Archiven der Geschichte. So sagte der deutsche Finanzminister Peer Steinbrück vor kurzem, Marx' Theorien könnten unter Umständen auch für die Krisen von heute relevant sein. Der französische Präsident Nicholas Sarkozy ließ beim Blättern durch Das Kapital fotografieren. Und der deutsche Filmemacher Alexander Kluge will sogar Das Kapital verfilmen.

Wir sollten uns ins Gedächtnis rufen, dass es schon früher dramatische Phasen der Globalisierung gab. Und ebenso wie bei früheren Globalisierungsschüben waren auch das Ende des 20. und der Beginn des 21. Jahrhunderts durch ein hohes Innovationstempo geprägt. Mit diesen Innovationen stieg vielerorts die Lebensqualität; der Wohlstand mehrte sich und breitete sich aus. Die weltweite Überwindung der Armut erschien zu Beginn des 21. Jahrhunderts greifbarer als je zuvor in der Geschichte. Die Gesamtbilanz dieser Globalisierungsepoche sieht so schlecht nicht aus. Doch vielleicht gab es mehr Innovation, als wir bewältigen konnten.

Auch frühere Wellen der Globalisierung wurden von der besonderen Dynamik eines bestimmten Wirtschaftssektors in Gang gesetzt, der sich jeweils durch einen außergewöhnlich hohen Innovationsgrad auszeichnete. Der Hinweis auf Karl Marx kann hier insoweit von Nutzen sein, als Marx unsere Aufmerksamkeit auf große Strukturbrüche lenkt. Wirtschaftshistoriker haben für die vergangenen 200 Jahre zwei solcher großen Brüche im Bereich der Wirtschaftsaktivität und der Beschäftigung identifiziert: erstens den Wandel von der Landwirtschaft hin zum produzierenden Gewerbe im 19. Jahrhundert, und zweitens einen neuen Schritt weg vom nunmehr ,alten“ produzierenden Gewerbe hin zu Dienstleistungen im 20. Jahrhundert. In beiden Fällen war es eine Krise, die den dramatischen Wandlungsprozess hin zu einem neuen Entwicklungsmodell in Gang brachte.

Die Expansion im 18. Jahrhundert wurde durch große Produktivitätssteigerungen in der Landwirtschaft vorangetrieben. Sie führten während der sogenannten Ersten Industriellen Revolution zu einem Anstieg der Kaufkraft und des privaten Konsums. In der zweiten Hälfte des 19. Jahrhunderts lösten technische Innovationen insbesondere im Eisen- und Stahlbereich dann eine Revolution im Verkehrswesen aus - allen voran die Dampfmaschine, aber auch Schiffsrümpfe aus Eisen.

Marx war ohne Zweifel ein scharfsichtiger Analytiker der Globalisierung im 19. Jahrhundert. 1848, also in einem Jahr der Umwälzungen, schrieb er im Коттиnistischen Manifest: „An die Stelle der alten lokalen und nationalen Selbstgenüg- 
samkeit und Abgeschlossenheit tritt ein allseitiger Verkehr, eine allseitige Abhängigkeit der Nationen voneinander.“

In den 1840er Jahren kam es nach Ernteausfällen in vielen Regionen zur letzten großen Hungersnot im traditionellen, ländlichen Europa. Diese Ernteausfälle waren massiv genug, dass es nicht nur für die Bauern, sondern für die gesamte Bevölkerung ökonomisch rapide bergab ging. Als die Krise überstanden war, wandten sich viele Landwirte anderen Erwerbsformen zu. Damit lösten sie in den Kernbranchen der ersten Industriellen Revolution einen starken Aufschwung aus.

Die Große Depression der 1930er Jahre war der Agrarkrise der 1840er Jahre ähnlich, was ihre transformatorische Wirkung auf wirtschaftliche Strukturen anging. Kurzfristig schien auch sie verheerend. Doch die wachsende Arbeitslosigkeit in der Industrie drängte die Arbeiter in neue Berufe. Fachkenntnisse und Bildung rückten nun in den Vordergrund, was wiederum die Dienstleistungsgesellschaft des 20. Jahrhunderts vorantrieb.

Die alten Wirtschaftszweige hörten in diesen Phasen wirtschaftlichen Wandels nicht auf zu existieren. Zwar mussten viele einzelne Unternehmen aufgeben. Doch insgesamt wurden die betroffenen Branchen lediglich um ein vielfaches effizienter. So sind die USA ein bedeutender Agrarproduzent und -exporteur geblieben, obwohl die Zahl der Landwirte auf einen verschwindend kleinen Anteil an der Gesamtbevölkerung geschrumpft ist. Zudem sind die Vereinigten Staaten, wenn auch nur knapp, noch immer der größte Industrieproduzent der Welt - aller Kritik an der Deindustrialisierung und am Verlust der relativen Wettbewerbsfähigkeit seit den 1970er und 80er Jahren zum Trotz.

Die Kreditklemme des Jahres 2008 ist ein Wendepunkt. Sie hat ernsthafte Schwächen des Finanzsektors offengelegt, der in den vergangenen Jahrzehnten die wesentliche Triebkraft für das Wirtschaftswachstum war. Zwei Innovationen spielten für die Dynamik des Finanzsystems in dieser Zeit die Hauptrolle: einmal die Verbriefung (securitisation), die es möglich machte, ganz unterschiedliche Eigentumsrechte und finanzielle Forderungen in homogene - und dadurch leicht handelbare - Wertpapierformate zu verpacken; diese konnten dann immer weiter verkauft werden. Die zweite maßgebliche Innovation war die Anwendung des Versicherungsprinzips auf diese Papiere. Credit Default Swaps boten auf der einen Seite für den Käufer ein großes $\mathrm{Ma} ß$ an Sicherheit, aber für den Anbieter ein fast unbegrenztes Risiko, das so groß war, dass nur wirklich sehr große und als stabil anerkannte Institutionen diesen Schutz anbieten konnten. Das Resultat 
war eine Bündelung sämtlicher Risiken in gewaltigen Unternehmen - von denen man annahm, sie seien einfach zu groß und zu kompliziert, um zu scheitern.

Durch eine wiederholte Aufspaltung, Neugliederung und Wiederverpackung solcher verbriefter Wertpapiere wurde es möglich, dass Anzahl und Wert der Schuldtitel den Wert der ursprünglich zugrunde gelegten Eigentumsrechte und Forderungen bei weitem übertraf. Doch man ging davon aus, dass die Verteilung des Risikos auf viele, wiederholt miteinander handelnde Partner risikomindernd wirken würde.

Im Nachhinein hat sich allerdings herausgestellt, dass gerade diese übergroße Komplexität bereits beim ersten großen Verlust einen Schatten auf die gesamte Finanzbranche wirft. Denn schon an diesem Punkt wird offensichtlich: Wie die Risiken verteilt und wo sie versteckt sind, ist unklar und bedarf der juristischen Klärung.

Versagt hat insbesondere die Bankenaufsicht, aber nicht weil sie nicht groß oder komplex genug gewesen sei, sondern weil sie die Anhäufung der Risiken nicht sehen oder beurteilen konnte. Und dies gilt sowohl für die externe Aufsicht als auch für das interne Risikomanagement der Finanzinstitute. Besonders gravierend waren die Mängel ausgerechnet in den Ländern mit den am weitesten entwickelten Finanzsektoren - den USA, Großbritannien, Frankreich und der Schweiz. Italien und Spanien hingegen, die zwar dynamische Bankensysteme hatten, im Allgemeinen aber immer noch als rückständig galten, funktionierte die Aufsicht deutlich besser.

Für die Anpassung an das neue Umfeld sind nun im Grunde zwei gegensätzliche Szenarien denkbar. Da wäre zunächst die politische Antwort auf das Scheitern des Experiments mit dem Risiko: mehr Regulierung. Angesichts der landwirtschaftlichen Krise im 19. Jahrhundert riefen die Agrarier, vor allem die Gutsbesitzer im ländlichen Osten Europas, nach einer stärkeren Regulierung der Agrarpreise. Im Chaos der 1930er Jahre erhoffte man sich durch erzwungene Unternehmenszusammenschlüsse, Kartelle und staatliche Aufsicht Wege aus der industriellen Krise. Solche Interventionen führten aber zu immer höheren Erwartungen an die Politik - und zu Enttäuschungen und Spannungen, als die politischen Versprechen nicht eingehalten wurden. Auch die trugen bei zur gewaltigen Krise des Staates und der Demokratie. Heute kommen viele neue Initiativen zur Verbesserung der Regulierung, zur Disziplinierung der Hedge-Fonds, zur Neuordnung der Einbindung von den Rating-Agenturen in die gesetzlich vorgeschriebenen Kapitalquoten, oder zur Mäßigung des Immobiliengeschäfts. Aber 
die Staaten, die neu und besser regulieren wollen, stehen vor einem großen und noch nicht gelösten Problem: Solche Regelungen können nur in internationaler Zusammenarbeit errichtet werden, aber gerade in einer großen Krise wird die internationale Kooperation immer schwieriger.

Die bessere Antwort auf Strukturbrüche war schon immer der technologische Wandel. Der Einsatz neuer Geräte und Technologien hatte stets zur Folge, dass Landwirtschaft und Industrie sehr viel produktiver wurden. Diese mussten nicht mehr so viele Menschen beschäftigen wie zuvor und wurden gesamtwirtschaftlich weniger verletzbar.

Auch für die Probleme der Banken dürften Innovationen die langfristige Lösung sein. Regulierung und Aufsicht werden künftig so funktionieren, dass sie in erster Linie die Reaktionen auf hypothetische Bedrohungsszenarien überprüfen etwa den Konkurs eines wichtigen Geschäftspartners oder geopolitische Umbrüche. Das ist im Gefolge der Kreditklemme bereits geschehen. Der Trend wird überdies hin zu größerer Schlichtheit gehen: weg von den Strukturen der weltumspannenden Universalbanken und Allfinanzanbieter, wie sie insbesondere die amerikanische Citigroup verkörperte, und hin zu schlichteren und transparenteren Organisationsformen.

Um Interessenkonflikte innerhalb einer Bank (zum Beispiel zwischen Beratertätigkeit und Vermögensverwaltung) auszuschließen oder mindestens zu minimieren, können künftig Algorithmen eingesetzt werden, die finanzielle Transaktionen zulassen oder verbieten. So wie im Börsengeschäft heute fast kein Parketthandel mehr stattfindet, können und werden viele Bankfunktionen von Maschinen übernommen werden. Interagierende Softwaresysteme werden die Vermittlung von Finanzgeschäften nach und nach übernehmen. Der Tätigkeit von „Halbstarken, Pyromanen und Gangstern“ (Franz Müntefering) wird so ein Ende gesetzt. ${ }^{3}$ Es sind aber nicht nur die Finanzinstitute, die innovieren müssen. In den vorigen historischen Transformationsprozessen war es oft ein Ändern des Konsumentenverhaltens, das zu durchgreifenden Neuerungen geführt hat. Um eine neue Dynamik zu entfachen, brauchen wir also besser informierte Kunden, die sich wie mündige Bürger im Finanzsystem bewegen können und die nicht zu Opfern von skrupellosen Marktmanipulatoren werden. Im Moment gibt es viel zu viele Defizite hinsichtlich ganz einfacher Kenntnisse über Geld, Rendite und Gewinn oder Verlust. 
Wie in früheren Phasen wirtschaftlichen Wandels werden all jene, die in der Finanzindustrie arbeiten, überzeugende Argumente vorzubringen suchen, wonach die menschliche Komponente für ihr Geschäft unverzichtbar sei - so wie einst vorausgesagt wurde, dass Erntemaschinen eine Qualitätsminderung des Getreides bewirken müssten, weil dieses nicht mehr an Ort und Stelle mit menschlichem Auge geprüft würde. Britische Eisenbahner beharrten lange darauf, dass zwei Lokomotivführer nötig seien, um die Sicherheit eines Zuges zu gewährleisten; heute werden in einigen öffentlichen Verkehrssystemen führerlose Züge eingesetzt. Die Produktion in modernen Automobilfabriken wird überwiegend von Robotern gemeistert. Die vielen Analysten und sogenannten unabhängige Berater, die jetzt als Mittelinstanzen zwischen Sparer oder Anleger und Kreditbezieher eingeschaltet werden, vermehren die Komplexität, ohne den Effizienz der Kapitalallokation bedeutend zu steigern. Vieles könnte auch hier automatisiert werden.

Die jüngste Anwendung der Psychologie auf die Wirtschaft hat gezeigt, wie irrational viele menschliche Entscheidungen sind. Die Geschichte der Kreditkrise war häufig genug die vertraute Mär vom charakterschwachen Individuum oder vom schurkischen Händler, wie wir sie von früheren Finanzkrisen her kennen. Mit dem Einsatz der Computertechnologie lässt sich das Potenzial für menschliches Versagen weitgehend vermindern. Wenn ein Computer es zulässt, dass Fonds selbst verwaltet werden, braucht man keinem Bernard Madoff zu trauen. Und werden 2009 in der Finanzindustrie weitere Stellen gestrichen, so könnte sich durchaus herausstellen, dass ein abgespeckter Finanzsektor besser funktioniert. Zumindest auf längere Sicht sind daher auch schwere und schmerzliche Strukturbrüche durchaus mit Zuversicht zu bewerten.

\section{II.}

Das gewaltige Anschwellen des Finanzsektors ist die Antwort auf sogenannte globale Ungleichgewichte. ${ }^{4}$ Dies gilt in erster Linie für das Ungleichgewicht zwischen der niedrigen Sparquote in den USA und anderen angelsächsischen Ländern auf der einen - und der sehr hohen Sparquote in China und anderen asiatischen Schwellenländern auf der anderen Seite. In den letzten acht Jahren wurden die asiatischen Währungsreserven massiv aufgebläht. Über amerikanische Staatsschuldpapiere gelangte jedoch ein Teil des Geldes in das Finanzsys- 
tem, um dann in den USA den Kauf von Immobilien und Autos sowie den allgemeinen Konsum zu finanzieren. Die armen Chinesen liehen den reichen Amerikanern das Geld, damit diese billige Waren aus China kaufen und Arbeitsplätze für die chinesische Bevölkerung garantieren konnten.

Zwei überraschende Schlussfolgerungen haben sich aus der aktuellen Diskussion ergeben, aber erst über eine davon ist man sich inzwischen vollständig im Klaren. Erstens bedarf es umfassender Maßnahmen des öffentlichen Sektors. Zweitens sind solche Maßnahmen hochkompliziert, weil in einer globalisierten Welt grenzübergreifende Hilfen erforderlich sind.

Zunächst wurden privatwirtschaftliche Lösungskonzepte ausprobiert; sie sind jedoch in atemberaubend kurzer Zeit gescheitert. Über dieses Scheitern mag nun die Aussicht hinwegtrösten, dass eine wirklich schwere Krise reinigend wirkt. Doch es ist selten, dass die Politik den Mut aufbringt, den gesamten Reinigungsprozess durchzuhalten.

US-Finanzminister Hank Paulson, der von der stärksten US-Investmentbank Goldman Sachs in die Regierung gewechselt war, spekulierte auf eine schnelle Selbstreinigung des Marktes, als er die Pleite von Lehman Brothers geschehen ließ. Zu diesem Zeitpunkt argumentierte er, die USA dürften keine „bailoutKultur" zulassen. Die strikte Weigerung der Regierung sollte ein Zeichen setzen: Die größten Teile der US-Wirtschaft seien im Wesentlichen gesund und die amerikanischen Finanzmärkte ausreichend hoch entwickelt, um gesunde Geschäftspraktiken von schlechten unterscheiden zu können.

Auch zur Zeit der Großen Depression amtierte in Amerika ein Titan des Bankensektors als Finanzminister: Andrew Mellon. Seine unmittelbare Schlussfolgerung aus der Börsenpanik von 1929 hat traurige Berühmtheit erlangt: „Arbeitsplätze liquidieren, Vorräte liquidieren, die Farmer liquidieren, Immobilien liquidieren [...] die Fäulnis aus dem System waschen. “5

Schon jetzt ist klar, dass sich Paulsons hoch riskante Wette vom September 2008 nicht ausgezahlt hat - ebenso wenig wie Mellons Kalkül im Jahr 1929. Im Gegenteil: Die unterlassene Hilfeleistung hat andere Rettungsaktionen erforderlich gemacht - etwa bei AIG in den USA und bei HBOS in Großbritannien. Und das dürfte noch längst nicht alles gewesen sein. Schwarze Listen zirkulierten, welche

5 Zitiert nach Bradford de Long, J.: Fiscal Policy in the Shadow of the Great Depression, in: Bordo, M.D./Goldin, C./White, E.N. (Hg.): The Defining Moment: The Great Depression and the American Economy in the Twentieth Century, Chicago, 1998, 67-85, hier 75. 
Institute als nächste zu Fall kommen - oder zu Fall gebracht werden könnten. Und für die Staaten, in denen diese Banken beheimatet waren, zirkulierten ähnliche Listen: erst Island, dann Irland, dann Großbritannien.

In einem derart labilen Finanzsystem sind nur noch Institutionen mit mehr oder weniger unbegrenzten Ressourcen in der Lage, sich gegen den Strom zu stemmen. Das könnten Selbsthilfeorganisationen sein, zum Beispiel ein Kartell mächtiger Banken. Tatsächlich hat das US-Finanzministerium am 14. September 2008 versucht, ein solches Kartell zu schmieden.

Doch in einem Klima extremer Verunsicherung reicht Selbsthilfe allein nicht aus. Die Regierungen und Notenbanken müssen eingreifen, denn nur sie können mit dem nötigen Gewicht und schnell genug reagieren. Nur Regierung und Fed gemeinsam konnten den Hypothekenriesen Fannie Mae und Freddie Mac rechtzeitig zur Hilfe kommen, um gleich darauf AIG zu retten und dann auch noch die krisengeschüttelten Großbanken Citigroup und Bank of America zu stützen.

Die Banken selbst sind nun in einer Zwangslage. Sie haben zum einen die Möglichkeit, ihre Bilanzen gesundzuschrumpfen. Das führt zu einer Verringerung der Kreditmenge und einem immer stärkeren Druck auf die Unternehmen, die ihrerseits ihre Schulden rapide abbauen müssten. Damit drohen Insolvenzen und ein weiteres Absinken der gesamten Kreditwürdigkeit. Das Resultat ist ein Teufelskreis: Die Bewertung der bestehenden Kreditrisiken verschlechtert sich, und die Banken werden zu neuen Abschreibungen gezwungen, wodurch sich die Eigenkapitalbasis weiter verringert - und erneut Kredite abgebaut werden müssen. Dies ist der klassische Prozess der Schulden-Deflation (debt-deflation), wie ihn der US-Ökonom Irving Fisher bereits in den 1930er Jahren analysiert hat. ${ }^{6}$

Die zweite rettende Möglichkeit besteht in einer Rekapitalisierung der Banken. Diese Aufgabe könnte der verunsicherte Markt allerdings nicht stemmen, dazu wäre nur der allein kreditwürdige Staat in der Lage. Eine Variante, die eher als marktkonform gilt, ist die Übernahme schlechter Kredite durch eine oder mehrere sogenannte bad banks. Diese Option wurde bereits während der Weltwirtschaftskrise der Zwischenkriegszeit erprobt. In Deutschland schuf man damals zwei solcher staatlichen Institute, die Tilgungskasse für Gewerbliche Kredite AG und die Deutsche Finanzierungsinstitut AG, die ausfallgefährdete Werte zu verbilligten Amortisationsraten übernahmen (jeweils $1 \%$ und $3 \%$ ). Sie spielten bei der Überwindung der Krise aber eine eher untergeordnete Rolle. Eine derartige 
bad bank war auch Bestandteil des ursprünglichen Troubled Assets Recovery Program (TARP) von US-Finanzminister Paulson. Doch es stellte sich schnell heraus, dass die Identifizierung und Bewertung der problematischen Vermögenswerte (troubled assets) allzu kompliziert und langwierig sein würde. Stattdessen verfiel man auf die einfachere Lösung einer Rekapitalisierung der Banken mit öffentlichem Geld. In einer neuen Wendung kommen viele Stimmen - darunter auch die von Federal Reserve Chairman Ben Bernanke - jetzt zurück auf die erste Lösung, die des Aufkaufens der problematischen Posten bei den Finanzinstituten durch den Staat.

Warum aber sollten nur die Banken von den Mitteln des Staates profitieren? Die Frage schien nur zu berechtigt. Das Prinzip der Stützung notleidender Unternehmen wird also immer weiter ausgedehnt - auf Automobilhersteller, dann auf Zulieferer von Automobilteilen, dann auf andere wichtige oder stimmkräftige Interessengruppen. Europäische Regierungen, vor allem die französische, denken über nationale Beteiligungsfonds nach, die neue Industriestrategien durchführen sollen.

Man dehnte also das Prinzip weiter aus, eine breiter angelegte und gerechtere Verteilungspolitik sollte die Konjunktur ankurbeln, kurz: Man suchte nach einer Lösung im keynesianischen Stil. Die Fiskalpolitik sollte helfen, wo die Geldpolitik gescheitert war - obwohl viele Wirtschaftswissenschaftler und -historiker die Wirksamkeit solcher konjunkturstützenden Maßnahmen bezweifeln. $\mathrm{Zu}$ diesen Skeptikern zählt auch Christina Romer, die neue Wirtschaftsberaterin im Weißen Haus (Vorsitzende des Council of Economic Advisers). ${ }^{7}$

Im Verlauf der Krise kam es demnach zu einer Eskalation der Antworten. Am Anfang stand die Liquiditätszufuhr, die schon im September 2008 in gewaltigem Umfang von den Zentralbanken eingesetzt wurde. Diese Hilfe schlug aber nicht sofort an. Es kann sein, dass sie tatsächlich Wirkung zeigt - doch ein solcher Eingriff lässt sich mit einem Kurswechsel bei großen Ozeanschiffen vergleichen, wo das Umschwenken des Ruders nicht sofort die Richtung ändert. Dann ist die Versuchung groß, andere Lösungen auszuprobieren. Es ist so, als ob der Computer einfriert, und man sofort hektisch auf alle möglichen Tasten drückt. Meistens haben solche Manöver keinen Erfolg. Die Lösungen sind teuer. In diesem Jahr rechnet man damit, dass sich die öffentliche Neuverschuldung weltweit um 3.000

7 Siehe Bordo, M.D./Goldin, C./White, E.N. (Hg.): The Defining Moment: The Great Depression and the American Economy in the Twentieth Century, Chicago, 1998. 
Mrd. US\$ erhöht, also um das dreifache der Zunahme im Jahr 2008. Wer soll nun diese Schulden finanzieren?

Eine zweite Frage wäre, welche Art von Regierung der Aufgabe gewachsen ist und das sind gewiss nicht alle. Die Regierungen mittelgroßer europäischer Länder etwa können womöglich mittelgroße europäische Institute retten. Doch im Fall der wirklich großen Finanzkonglomerate, die den Kern des weltweiten Finanzsystems bilden, haben vermutlich nur zwei Länder die nötige Durchschlagskraft: die USA und China.

In Europa zeigen sich mit Blick auf eine mögliche keynesianische Fiskalpolitik tiefe Gräben. So haben etwa Italien, Griechenland und Portugal eine zu hohe Staatsverschuldung, um sich keynesianische Lösungen leisten zu können. In Großbritannien, Irland oder Spanien ist die Quote der Staatsschulden zwar geringer. Aber dafür hat dort die private Verschuldung derart monströse Ausmaße angenommen, dass diese Länder kaum in der Lage sind, private schlicht durch staatliche Schulden zu ersetzen. Ein Ausdehnen der staatlichen Aktivität als allgemein empfohlenes Rettungsprinzip in der Finanzkrise wird sich also als politisches Dynamit für den bisher so erfolgreich laufenden Prozess der europäischen Integration erweisen. Einige Länder - darunter Deutschland - werden dazu fähig sein, die Mehrzahl aber nicht. Die EU selbst, mit Ausgaben, die nur knapp über $1 \%$ des europäischen BSP liegen, ist bei dieser Aufgabe impotent. Diese unterschiedlichen Spielräume werden sich wie tiefe Gräben im europäischen Gebäude auswirken.

Auch hier verblüfft die historische Parallele: Unter den vergleichbaren Umständen der finanziellen Kernschmelze von 1931 gab es ebenfalls nur eine sehr begrenzte Zahl von Regierungen, die tatsächlich etwas ausrichten konnten. Im Falle Deutschlands hat vor dreißig Jahren Knut Borchardt seine keynesianischen Kollegen mit dem Beweis erschreckt, wie begrenzt die Handlungsspielräume für Reichskanzler Heinrich Brüning in der Krise der Weimarer Republik waren. ${ }^{8}$ Auch anderswo waren die Probleme erschreckend groß, die Antworten enttäuschend hilflos. Die alte wirtschaftliche Supermacht Großbritannien war zu erschöpft, um irgendjemandem außer sich selbst zu helfen. Und ein Großteil der weltweiten Reserven war bereits in den USA angehäuft. Damals waren nur die USA wirklich handlungsfähig.

8 Borchardt, K.: Zwangslagen und Handlungsspielräume in der großen Wirtschaftskrise der frühen dreißiger Jahre, in: Bayerische Akademie der Wissenschaften (Hg.): Jahrbuch 1979, München, 1979, 85-132. 
Als einzig plausibles Szenario für einen Ausweg aus der Großen Depression wäre damit, wie Charles Kindleberger ausgeführt hat, ein Eingreifen der USA geblieben. ${ }^{9}$ Nun gab es damals für die Amerikaner eine Menge überzeugender Gründe, sich die Last einer weltweiten Rettungsaktion gar nicht erst aufzubürden. Mehr Geld nach Europa zu schicken, hätte so ausgesehen, als werfe man es zum Fenster hinaus. Und hatten nicht die Europäer jenen Weltkrieg begonnen, der Ursprung und Quelle der Finanzmisere war? Wirtschaftlich gesehen und bei langfristiger Betrachtungsweise wäre ein solcher Schritt überaus sinnvoll gewesen. Politisch freilich war er eine Totgeburt ohne jede Aussicht auf kurzfristigen Gewinn.

Das Amerika dieses Jahrhunderts ist China. Die frühen Stadien der Kreditverknappung wurden scheinbar so schmerzlos bewältigt, weil Staatsfonds aus dem Nahen Osten, vor allem aber aus China bereit waren, in die Bresche zu springen. Sie versorgten die verschuldeten amerikanischen und europäischen Institute mit frischem Geld. Anders als die großen Finanzhäuser in Europa und den USA hatten die asiatischen Banken am sub-prime-Risiko so gut wie nicht beteiligt.

Der Schlüsselmoment der aktuellen Ereigniskette war die Weigerung der chinesischen SWF China Investment Co., ihre Sondierung eines Kaufs von Lehman Brothers weiterzuführen. Der Rückzieher von CIC wird irgendwann als Punkt gelten, an dem die Geschichte eine andere Richtung hätte nehmen können.

Nun wird es für den Rückzug der Chinesen eine Menge Gründe gegeben haben. Die Logik klingt jedenfalls ähnlich wie im Fall der Amerikaner 1931. Einige der Argumente, die derzeit in Peking kursieren, sind durchaus vernünftig. Es gibt eine Menge Unsicherheiten, und die Staatsfonds könnten sehr viel Geld verlieren. Auch der CIC hätte ein Kauf der schwer angeschlagenen US-Investmentbank zunächst Verluste beschert. Zudem spielen Emotionen in der Debatte eine Rolle: Könnte 2008 nicht die Rache Chinas für das amerikanische Missmanagement der Ostasienkrise von 1997/98 sein?

Es wird sich bald zeigen, welches Interesse China wirklich am Überleben der globalisierten Weltwirtschaft hat. Wie 1931 sprechen alle politischen Argumente scheinbar gegen einen rettenden Eingriff. Nur wer weitsichtig ist, wird erkennen, dass die wirtschaftlichen Argumente für eine Rettung zwingend sind. Und wie in den 1930er Jahren werden Versuche gemacht werden - wie jetzt von Seite des neuen amerikanischen Finanzministers Timothy Geithner, der China als „Wäh-

9 Kindleberger, C.: The World in Depression, Berkeley, CA, 1973. 
rungsmanipulator“ denunzierte - die Schuld für die Krise dem großen Gläubigerstaat zuzuweisen, was natürlich dramatisch die Hilfsbereitschaft dieses Gläubigers beeinträchtigt.

Neue Formen der Ordnung, neue Technologien oder auch eine erneute Umwälzung der geopolitischen Verhältnisse mögen dafür sorgen, dass das es wieder Wachstum gibt. Auch hier kann man zumindest auf längere Sicht optimistisch sein - selbst in einem Europa, das sich bisweilen als überaltert und stagnierend empfindet. Aber werden wir unsere eigene Rückkehr zum Wachstum blockieren, werden wir uns abschotten von den Möglichkeiten einer neuen Dynamik?

\section{III.}

Es wird immer wieder behauptet, dass die Globalisierung - das Zusammenwachsen der Welt durch große Ströme von Waren, Kapital und auch Menschen irreversibel sei. Wie ein Mantra der Selbstberuhigung hat sich diese Behauptung über die vergangenen Jahrzehnte hinweg gehalten. Doch sie ist historisch nicht zu halten. Globalisierung ist nichts Neues, und es gibt in diesem Zusammenhang auch nichts, was unaufhaltbar wäre. Die weltwirtschaftliche Integration im 18. Jahrhundert, angetrieben von Zucker und Kaffee, provozierte Rückschläge gegen das englische und das französische Imperium, die diese Art der Globalisierung betrieben. Die Kriege der Französischen Revolution und der Napoleonischen Ära lenkten Handelsströme um und verlagerten die Produktion. Die Globalisierung im 19. Jahrhundert, mit ihrer tiefen Integration durch das Dampfschiff, das Telegramm und die Massenmigration, endete mit dem Ersten Weltkrieg. Nein, die Globalisierung ist nicht unumkehrbar, genauso wenig wie sie neu ist.

Der heutige Rückschlag gegen die Globalisierung wird von zwei Kräften vorangetrieben: praktische, inteteressengetriebene Reaktionen auf der einen Seite, und tief empfundene moralistische Argumente auf der anderen. Einzeln und für sich genommen blieben sie wohl oberflächlich und wirkungslos, doch zusammen erzeugen sie eine gewaltige Wirkung.

Um mit der praktischen Seite zu beginnen: Die gegenwärtige Krise wird zu einer steigenden Arbeitslosigkeit führen. Die Suche nach einer Bekämpfung der Arbeitslosigkeit führt unausweichlich zur Protektion, und tatsächlich sind bereits etliche bekümmernde Zeichen einer solchen Reaktion zu sehen, trotz vieler beruhigender Worte über den Wert der internationalen Offenheit.

Im November 2008 gelobten die Delegierten des G-20-Wirtschaftsgipfels in einer Erklärung, dass sie zwölf Monate lang „davon absehen, neue Barrieren 
gegen Investitionen oder den Handel mit Waren und Dienstleistungen“" aufzustellen. Doch binnen weniger Tage erließ Russland neue Zölle auf Automobile und ließ den Rubel abwerten, und Indien erließ Zölle auf Eisen- und Stahlerzeugnisse. China senkte außerdem den Wert des Renminbi, um seine schwächelnden Exporte zu stützen. In der Eurozone wird die scharfe Abwertung des britischen Pfundes wohl ebenfalls als eine gezielte Aktion wahrgenommen werden, als Teil eines Währungskrieges. Der neue Kongress der Vereinigten Staaten ist offener für die Handelsprotektion als der alte, und es erscheint unwahrscheinlich, dass Präsident Obama entsprechende Wünsche blockiert. Weiterhin gibt es eine dramatische Zuwendung zu einer Denkrichtung, die man als neo-keynesianischen Konsens bezeichnen könnte: dass hohe zusätzliche Staatsausgaben notwendig sind, um die Konjunktur zu retten, und dass große Staatsschulden gebraucht werden, um die Kreditmärkte zu stabilisieren, weil der Staat in extrem nervösen Zeiten der einzig verlässliche Schuldner ist.

Was passiert, wenn der Staat diese Erwartungen nicht erfüllen kann? Wenn die Defizite steigen und dennoch eine dramatische Wendung ausbleibt? Dann setzt eine neue und gefährliche Ernüchterung ein. Der Markt hat versagt, aber auch der Staat.

Historisch betrachtet waren Rückschläge gegen die Globalisierung von moralischer Entrüstung über die Verderbtheit des Handels über große Distanzen getrieben - und oft religiös inspiriert. Die sich ausweitende Welt der Renaissance inspirierte den italienischen Bußprediger Girolamo Savonarola zu einer derartigen Reaktion, und seine Anhänger veranstalteten „Fegefeuer der Eitelkeiten“, in welchen sie die Auswüchse des Florentinischen Handels mit Luxusgütern den Flammen überließen. Einen Nachahmer nördlich der Alpen fand Savonarola in Martin Luther, der Traktate gegen den Fernhandel schrieb, in denen er vom Heiligen Paulus ausging und dessen Standpunkt „Der geytz is eyn wurtzel alles ubels" (wobei das Wort „Geiz“ im Frühneuhochdeutschen als „Gier“ zu verstehen ist).

Globale Integration, globale Gewalt und eine Krise der Werte - diese Dinge sind, historisch gesehen, eng miteinander verbunden. Es sollte uns nicht merkwürdig vorkommen, wenn wir ihrem Zusammenspiel in unserem eigenen Zeitalter der Globalisierung begegnen. Doch in unserem Fall wird die Reaktion besonders gewaltig ausfallen, was an den vornehmlich finanziellen Ursachen der Krise liegt. 
Die Antworten und Verschreibungen unserer Ökonomen sind die Lösungen, die für das 20. Jahrhundert entwickelt wurden. Doch gibt es auch viel ältere Antworten auf dieses Problem. Deflation produziert radikalen Antikapitalismus und Forderungen nach einer Löschung der Schulden. Die Ablehnung der Marktwirtschaft nimmt oft die Form einer spezifischen Verdammung der Schulden und der Schuldinstrumente an.

Der saudische Geistliche Großmufti Abdelaziz Al al-Sheikh vertrat die Ansicht, dass die Ursache von Wirtschaftskrisen der Schuldzins an sich sei, und dass das Scharia-Prinzip der Risikoverteilung das Problem beseitigen werde. Das ist eine dieser sehr alten Antworten. Das Alte Testament empfahl bekanntermaßen das turnusmäßige Löschen der Schuld, alle 49 Jahre. Die Kirche des Mittelalters kämpfte gegen den Wucher. Im Vaterunser sagen wir: ,dimitte nobis debita nostra sicut et nos dimittimus debitoribus nostris “, „vergib uns unsere Schuld, wie auch wir vergeben unseren Schuldigern“. Der Begriff „Schuld“ bringt Moral und Geld miteinander in Beziehung.

Solche Antworten verstehen Schulden als die Ursache eines grundlegenden moralischen Defizits. Heute gibt es sehr viel mehr, sehr viel größere Schulden als im mittelalterlichen Europa. Verbraucher in entwickelten Industrieländern (und ganz besonders in den Vereinigten Staaten) besorgen sich Kredite, um einzukaufen.

Die theologische Interpretation der Moderne ist, dass wir voneinander leihen, immer mehr - aus einem verurteilungswürdigen Grund. Wir leihen, weil wir davon überzeugt sind, dass unser Nutzen, unser Genuss größer und wichtiger sind als der eines anderen Menschen. Wenn wir das blitzende Auto im Laden sehen oder die goldene Uhr beim Juwelier, die wir uns im Augenblick nicht leisten können, sind wir dennoch tief davon überzeugt, dass dieses Auto am besten von uns selber gefahren werden und die Uhr am besten unsere Hand zieren würde. Auf eine Weise nährt sich die Gier aus Stolz oder Selbstsucht.

Schon vor dem Sommer 2007 gab es einige Zeichen dafür, dass wir in ein neues Zeitalter eintreten, in dem die „Globalisierungsthese“ ein weiteres Mal zurückgerollt wird. Die Doha-Verhandlungsrunde zur Liberalisierung des Welthandels blieb stecken, weil ein tiefer Konflikt zwischen den neu zu Macht gekommenen Schwellenländern und den alten Industrieländern aufgebrochen war. In China und Russland nutzten autoritäre Staaten die Knappheit an Rohstoffen, um ihre weltpolitische Macht zu mehren. Eine weit verbreitete Skepsis über die Vorzüge des freien Kapitalverkehrs hat eingesetzt, und sogar der Internationale Wäh- 
rungsfonds ist heute sehr zögerlich in dieser Frage. Während die Wirtschaftslage sich verschlechtert, sorgen sich die Menschen in vielen Ländern über die Folgen der Einwanderung.

In dieser neuen Welt wird die Abgrenzung von Fremden wichtig. Wir werden auf das Lokale und das Traditionelle zurückkommen. Individuen sehen sich eher Risiken aus der Außenwelt ausgesetzt als Chancen. Länder sind bereit, Handelsund Währungskriege zu führen, und äußeren Einmischungen in ihre heimischen Geschäftsangelegenheiten zu widerstehen. Politiker konzentrieren sich darauf, das Handels- und Geldsystem neu zu gestalten, um die Balance zwischen politischer und ökonomischer Macht zu verändern. Die erweiterten, teuren Funktionen des Staates bedeuten auch, dass die Steuerzahler ein starkes Interesse daran haben, dass die Vorteile des neuen Keynesianismus nicht außerhalb der Landesgrenzen verwendet werden. Macht und Hegemonie gewinnen wieder an Bedeutung, weil politische Entscheidungsträger eher Nullsummenspiele sehen als die Vorteile der Zusammenarbeit. In dieser Welt neigen Konflikte dazu zu eskalieren und die Grundlage des Wohlstands und der internationalen Ordnung zu zerstören.

Kehren wir zurück zu Ödipus und zur Sophokleischen Tragik. Nachdem die Wissenschaftler nachgedacht und die Politiker Reden gehalten haben, rückt eine neue Interpretation in den Vordergrund: Es handelt sich nicht so sehr um ein leicht zu lösendes technisches, administratives oder bürokratisches Problem, sondern um einen ureigenen Wesenszug des Menschen. Hier sind es Schuld und Sünde. Im letzten Jahr eröffnete Klaus Schwab das jährliche Weltwirtschaftsforum in Davos mit den Worten: ,Jetzt kommt die Zeit, wo wir für unsere Sünden bezahlen müssen.“ In solchen Fällen sind die Meisten allerdings nicht geneigt, selbst das Büßergewand anzulegen, sondern wollen die Schuld in erster Linie bei den Anderen sehen. Doch die Vertreibung des Ödipus war für Theben keine Lösung: Es folgten weitere Gewalttaten, Mord, Selbstmord, Leichenschändung, kurzum: allgemeine Barbarei.

Erweiterte Fassung eines am 31. Januar 2009 vor der Berlin-Brandenburgischen Akademie der Wissenschaften gehaltenen Vortrages. 